\title{
A review of primer effects by murine pheromone signaling: pheromonal influences on reproductive conditions
}

\author{
Delia S. Shelton ${ }^{1}$
}

Received: 5 June 2016/ Accepted: 5 June 2016/Published online: 2 August 2016

(C) Japan Ethological Society and Springer Japan 2016

Great strides have been made in understanding murine behavior, development, communication, and the mechanisms that underlie specific phenomena. The popularity of mice as a model system has led to an explosion of literature on mouse behavior, development, sensory physiology, and associated mechanisms. Such a vast literature can be a daunting task for individuals to organize. The communication system of murid rodents is complex, involving behavioral, physiological, chemical, and neural pathways, and consequently an understanding of a breadth of literature besieged by intricate details is needed; a knowledge base that is organized by Sachiko Koyama, a mouse behavioral ecologist at Indiana University. Primer Effects by Murine Pheromone Signaling: Pheromonal influences on reproductive conditions is a succinct, pocket-sized book that helps make this vast literature accessible.

Koyama provides a brief overview of some of the historical and more recent forays into pheromone signaling in murid rodents. She selects key topics related to physiological and reproductive changes associated with primer effects. In seven chapters, she synthesizes major findings on primer effects on sperm motility, sperm density, mammary gland expansion, and their long-term effects on cognition. This presentation of primer effects on mouse pheromone signaling links multiple levels of analysis, spanning behavior, hormones and genes, with emphasis on genetic and neuro-hormonal pathways.

The book begins by summarizing the natural history of house mice, describing the ecological contexts where these

Delia S. Shelton

delsshel@indiana.edu

1 Psychological and Brain Sciences, Indiana University, 1101 E. 10th Street, Bloomington, IN 47405, USA primer effects occur. The heart of the book begins in Chapter 2, which introduces the morphological and physiological architecture of the mouse olfactory system. Chapters 3-6 provide a historical overview of primer effects, and then quickly transitions into a presentation of the exciting new findings related to primer effects. Primer Effects by Murine Pheromone Signaling details remarkable work that shows pheromones can influence neurogenesis and have long-lasting effects, i.e., affecting the cognition of future offspring. The book ends by thoughtfully inquiring about the influence of pheromone signaling on reproductive success, and then speculating about how primer effects may impact human reproductive physiology. Missing from this pocket-sized guide is a discussion on how pheromone signaling might be influenced by human-induced rapid environmental change. The effects of climate change on the sensory-physiological system are likely to be profound, as sensory systems are directly in contact with the changing environment.

Numerous diagrams and photographs enhance the text and make the material comprehendible to both experienced rodent experimenters and novices. The brevity and size of the book make it an accessible reference book, and are clear strengths of this pocket guide. The list of references that follow each chapter permit a curious reader to investigate topics of interest more deeply. Primer Effects by Murine Pheromone Signaling: Pheromonal influences on reproductive conditions will serve a quick go-to for those interested in a succinct introduction and overview of primer effects in a highly studied model system. 